\title{
Nonlinear normal modes in electrodynamic systems: A nonperturbative approach
}

\author{
A. V. Kudrin $*$ O. A. Kudrina, and E. Yu. Petrov \\ Department of Radiophysics, University of Nizhny Novgorod, \\ 23 Gagarin Ave., Nizhny Novgorod 603950, Russia
}

\begin{abstract}
We consider electromagnetic nonlinear normal modes in cylindrical cavity resonators filled with a nonlinear nondispersive medium. The key feature of the analysis is that exact analytical solutions of the nonlinear field equations are employed to study the mode properties in detail. Based on such a nonperturbative approach, we rigorously prove that the total energy of free nonlinear oscillations in a distributive conservative system, such as that considered in our work, can exactly coincide with the sum of energies of the normal modes of the system. This fact implies that the energy orthogonality property, which has so far been known to take place only for linear oscillations and fields, can also be observed in the nonlinear oscillatory system.
\end{abstract}

PACS numbers: 03.50.De, 02.30.Jr

\section{INTRODUCTION}

The concept of normal modes is a milestone in the theory of linear oscillating systems and has had a significant impact on all fields of physics [1 4]. As is known, a linear normal mode (LNM) is a free sinusoidal oscillation in a conservative dynamical system with constant parameters. In bounded distributed dynamical systems, an infinite but countable set of LNMs exists. In such systems, each LNM is characterized by its frequency and shape, and satisfies homogeneous partial differential equations (PDEs) of motion with given boundary conditions. A family of LNMs possesses the following important properties, which allows one to solve a whole set of problems related to the calculation of free and forced motions in a linear system.

1. Invariance. Each LNM can be excited independently of other LNMs by the specific choice of the initial conditions.

2. Completeness. An arbitrary oscillatory process in the system can be expressed as a superposition of LNMs.

3. Energy orthogonality. The total energy present in the system due to a free oscillatory process is the sum of the LNM energies.

Since nature is nonlinear, LNMs can only be regarded as very useful mathematical models describing actual oscillations of nonlinear systems in the weak-amplitude limit. However, the following question naturally arises: Do strongly nonlinear systems admit such specific motions that their properties allow one to consider them as nonlinear normal modes (NNMs), i.e., nonlinear generalizations of the LNMs of the underlying linear systems? An affirmative answer this question with respect to the lumped systems has been done in the seminal works of Lyapunov [5] and Rosenberg [6 8]. Rosenberg defined an NNM as a vibration in unison of the mechanical system, i.e., a synchronous oscillation during which all the displacements of the material points of the system

\footnotetext{
* kud@rf.unn.ru
}

reach their extreme values and pass through zero simultaneously. A definition of the NNM in an autonomous distributed system in terms of the dynamics on a twodimensional invariant manifold in phase space has been proposed by Shaw and Pierre [9]. Using the invariant manifold approach, they have also developed the technique of asymptotic series expansions for constructing NNMs for a rather wide class of nonlinear $1+1 \mathrm{D}$ autonomous systems. In the past decades, the concept of NNMs in mechanical systems has been studied extensively by a large number of workers (see, e.g., works 10 16] and references therein). At the same time, NNMs in electrodynamic systems remain poorly studied.

In this work, we present a nonperturbative approach to the concept of NNMs in an exactly integrable, nonlinear $2+1 \mathrm{D}$ electrodynamic system. It should be emphasized that all the forthcoming results are exact, i.e., no asymptotic expansions will be used. Our approach is based on the theory developed in [17, 18]. The nonlinear PDEs considered in [17, 18] and herein depend explicitly on the independent variable (radial coordinate) and can formally be regarded as $1+1 \mathrm{D}$ nonautonomous systems. Therefore, the approach proposed in [9], which is restricted to the autonomous systems, cannot be applied directly. In the present study, we define an NNM as follows: The nonlinear normal mode (NNM) of a bounded distributed conservative system is an oscillatory motion in which all of the field quantities oscillate periodically in time with the same constant period in the whole volume of the system. Each of the NNM fields must exactly satisfy the nonlinear PDEs of motion and the boundary conditions, and reduces to the corresponding LNM field in the weak-field limit. Note that a similar generalized definition of the NNM as an unnecessarily synchronous periodic motion in a mechanical system was proposed in [16].

In what follows, we construct the electromagnetic NNMs in cylindrical resonators filled with a nonlinear nondispersive medium and discuss their properties in detail. It will be shown that the considered NNMs, as their linear counterparts, exactly satisfy the first abovementioned property of invariance and, what is quite re- 
markable, the third (energy orthogonality) property.

\section{BASIC EQUATIONS}

Consider electromagnetic fields in a bounded cylindrical cavity of radius $a$ and height $L$. We assume that the $z$ axis of a cylindrical coordinate system $(r, \phi, z)$ is aligned with the cavity axis and limit ourselves to consideration of axisymmetric field oscillations, in which only the $E_{z}$ and $H_{\phi}$ components are nonzero. We will also assume that the cavity is filled with a nonlinear nondispersive medium in which the longitudinal component of the electric displacement can be represented as $D_{z}=D_{0}+\alpha^{-1} \epsilon_{0} \varepsilon_{1}\left[\exp \left(\alpha E_{z}\right)-1\right]$, where $D_{0}, \varepsilon_{1}$, and $\alpha$ are certain constants. Since even powers of $E_{z}$ are present in the series expansion of $D_{z}$, the medium does not possess a center of inversion. This is inherent in, e.g., uniaxial pyroelectric and ferroelectric crystals, provided that the $z$ axis is aligned with the crystallographic symmetry axis. With appropriately chosen constants $D_{0}$, $\varepsilon_{1}$, and $\alpha$, such an exponential constitutive relation correctly describes dielectric properties of actual media lacking a center of inversion in the case of weak nonlinearity where we can restrict ourselves to the quadratic (in $E_{z}$ ) correction term to the linear dependence of $D_{z}$ on $E_{z}$ (see [17, 18] for more details). Note that the model of a nonlinear distributed system presented herein and its generalizations were also discussed in works [19 23]. In this case, the Maxwell equations read

$$
\begin{array}{r}
\frac{\partial H}{\partial r}+\frac{1}{r} H=\varepsilon(E) \frac{\partial E}{\partial t}, \\
\frac{\partial E}{\partial r}=\mu_{0} \frac{\partial H}{\partial t},
\end{array}
$$

where $E \equiv E_{z}(r, t), H \equiv H_{\phi}(r, t)$, and

$$
\varepsilon(E) \equiv d D_{z} / d E=\epsilon_{0} \varepsilon_{1} \exp (\alpha E)
$$

An exact solution to the system of equations (1) and (2) can be written in implicit form as [18]

$$
\begin{aligned}
& E=\mathcal{E}\left(\rho e^{\alpha E / 2}, \tau+\alpha Z_{0} \rho H /\left(2 \varepsilon_{1}^{1 / 2}\right)\right) \\
& H=Z_{0}^{-1} \varepsilon_{1}^{1 / 2} e^{\alpha E / 2} \mathcal{H}\left(\rho e^{\alpha E / 2}, \tau+\alpha Z_{0} \rho H /\left(2 \varepsilon_{1}^{1 / 2}\right)\right)
\end{aligned}
$$

Hereafter, $\rho=r / a, \tau=t\left(\epsilon_{0} \varepsilon_{1} \mu_{0}\right)^{-1 / 2} / a$, and $Z_{0}=$ $\left(\mu_{0} / \epsilon_{0}\right)^{1 / 2}$. The functions $\mathcal{E}$ and $\mathcal{H}$ describe the electromagnetic field in a linear medium and satisfy the equations

$$
\frac{\partial^{2} \mathcal{E}}{\partial \rho^{2}}+\frac{1}{\rho} \frac{\partial \mathcal{E}}{\partial \rho}=\frac{\partial^{2} \mathcal{E}}{\partial \tau^{2}}
$$

and

$$
\frac{\partial \mathcal{E}}{\partial \rho}=\frac{\partial \mathcal{H}}{\partial \tau} .
$$

The energy conservation law in the considered nonlinear medium can easily be derived from the field equations (1) and (2). Multiplying (1) by $E$, and (2) by $H$, after some algebra we obtain

$$
\frac{\partial w}{\partial t}+\nabla \cdot \mathbf{S}=0
$$

with the energy density

$$
w=\epsilon_{0} \varepsilon_{1} \frac{(\alpha E-1) \exp (\alpha E)+1}{\alpha^{2}}+\mu_{0} \frac{H^{2}}{2}
$$

and the Poynting vector

$$
\mathbf{S}=-\mathbf{r}_{0} E H
$$

In the weak-field limit $(|\alpha E| \ll 1)$, expression $(7)$ reduces to the well-known textbook formula $w=\epsilon_{0} \varepsilon_{1} E^{2} / 2+$ $\mu_{0} H^{2} / 2$.

Based on formulas (4), the method proposed in [18] makes it possible to easily construct exact periodic solutions of the system of equations (1) and (2), starting from the LNMs which satisfy the linear wave equation (5). It is the purpose of the forthcoming analysis to demonstrate that the constructed solutions can actually be identified as NNMs of the considered electrodynamic systems.

\section{NONLINEAR NORMAL MODES IN A CYLINDRICAL CAVITY RESONATOR}

Analytical solution for the oscillations of the $E_{0 n 0}$ type in a circular cylindrical cavity with perfectly conducting walls and the nonlinear filling medium described by the dynamic permittivity (3) has been found in [18] and is given by

$$
\begin{aligned}
& E=A J_{0}\left(\kappa_{n} \rho e^{\alpha E / 2}\right) \cos \left(\kappa_{n} \theta\right), \\
& H=-A Z_{0}^{-1} \varepsilon_{1}^{1 / 2} e^{\alpha E / 2} J_{1}\left(\kappa_{n} \rho e^{\alpha E / 2}\right) \sin \left(\kappa_{n} \theta\right),
\end{aligned}
$$

where $A$ is an arbitrary amplitude factor, $J_{m}$ is a Bessel function of the first kind of order $m, \kappa_{n}$ is the $n$th positive root of the equation $J_{0}(\kappa)=0$, and $\theta=\tau+$ $\alpha Z_{0} \rho H /\left(2 \varepsilon_{1}^{1 / 2}\right)$. The electric and magnetic fields are described by the implicit functions $E(r, t)$ and $H(r, t)$ which are solutions of system (9) of two transcendental equations. These implicit functions exactly satisfy Maxwell equations (11) and (2), as well as the boundary conditions

$$
E(a, t)=0, \quad|E(0, t)|<\infty .
$$

The initial conditions can be obtained by substituting $\tau=0$ into formulas (9) to give

$$
E=A J_{0}\left(\kappa_{n} \rho e^{\alpha E / 2}\right)
$$

at $t=0$ and

$$
H(r, 0) \equiv 0
$$


For a sufficiently large index $n$ such that $n>n^{*}(\alpha)$, where $n^{*}$ is a certain integer, the functions $E(r, t)$ and $H(r, t)$ become ambiguous and solution (9), obtained without allowance for dispersion, becomes inapplicable [18]. For $n<n^{*}$, implicit functions $E$ and $H$ given by formulas (9) describe continuous periodic oscillations with the time period $T_{n}=2 \pi / \omega_{n}$, where $\omega_{n}=\kappa_{n}\left(\epsilon_{0} \varepsilon_{1} \mu_{0}\right)^{-1 / 2} a^{-1}$, satisfy the NNM definition formulated above, and correspond to the NNMs of the $E_{0 n 0}$ $\left(\mathrm{TM}_{0 n 0}\right)$ type in the cavity. Specifying the integer index $n$ and imposing the initial conditions (11) and (12), the motion of the system follows the exact solution (9) of the nonlinear boundary value problem for equations (11) and (2), i.e., no oscillations with indices differing from $n$ are excited. Hence, the considered NNMs satisfy the invariance property.

Let us now consider some important features of NNMs, which were not pointed out in our previous work [18]. First of all, the electric field in these modes does not oscillate in unison in the whole cavity volume, i.e., the amplitudes of the field at different spatial points can reach their extreme values and pass through zero at different instants of time. The same is true for the magnetic field. It is clearly seen in Fig. 3 in [18] that there are no synchronous oscillations at different spatial points in the $E_{020}$ mode discussed therein. In this respect, the considered electromagnetic NNMs differ from the well-known NNMs in lumped and $1+1 \mathrm{D}$ distributed mechanical systems [8, [9] and can be called "internally resonant" [15, 16]. It will be shown below that the degree of oscillation synchronism at different points of the cavity resonator depends on the shape of the cavity.

We now calculate the total energy $W$ stored in each NNM of the $E_{0 n 0}$ type. To this end, one should substitute the implicit functions $E$ and $H$ given by formulas (9) into (7) and integrate $w$ over the cavity volume:

$$
W=a^{2} \int_{0}^{L} \int_{0}^{2 \pi} \int_{0}^{1} w(\rho, \tau, \alpha) \rho d \rho d \phi d z .
$$

A remarkable result is that the quantity $W$ is independent of the nonlinearity parameter $\alpha$ and exactly coincides with the total energy $W_{0}^{(n)}$ of the corresponding linear $E_{0 n 0}$ mode in the cavity resonator filled with a linear medium that has the permittivity $\varepsilon=\epsilon_{0} \varepsilon_{1}=$ const. The rigorous proof of this fact is given in the Appendix. Note that the quantity $W_{0}^{(n)}$ is calculated analytically as

$$
\begin{aligned}
W_{0}^{(n)}= & \pi \epsilon_{0} \varepsilon_{1} a^{2} L \int_{0}^{1}\left(\mathcal{E}^{2}+\mathcal{H}^{2}\right) \rho d \rho= \\
= & \pi \epsilon_{0} \varepsilon_{1} a^{2} L A^{2}\left[\cos ^{2}\left(\kappa_{n} \tau\right) \int_{0}^{1} J_{0}^{2}\left(\kappa_{n} \rho\right) \rho d \rho+\right. \\
& \left.+\sin ^{2}\left(\kappa_{n} \tau\right) \int_{0}^{1} J_{1}^{2}\left(\kappa_{n} \rho\right) \rho d \rho\right]=
\end{aligned}
$$

$$
=\frac{\pi}{2} \epsilon_{0} \varepsilon_{1} a^{2} L A^{2} J_{1}^{2}\left(\kappa_{n}\right) .
$$

It is worth also nothing that the fundamental frequencies $\omega_{n}$ of the NNMs are independent of the field amplitude and the total energy, and coincide with the eigenfrequencies of the LNMs of the $E_{0 n 0}$ type in the underlying linear system. In the next section, we will show that the abovementioned notable features of the NNMs hold for another electromagnetic system described by equations (1) and (2).

\section{NONLINEAR NORMAL MODES IN A COAXIAL RESONATOR}

Assume that a coaxial cylindrical inner conductor of radius $b(0<b<a)$ is inserted inside the cavity considered in the previous section. The NNMs of the $E_{0 n 0}$ type in the nonlinear coaxial resonator can readily be constructed using formulas (4) from the corresponding LNMs in the linear resonator with a constant permittivity $\varepsilon=\epsilon_{0} \varepsilon_{1}(\alpha=0)$. The electric fields of the LNMs of the $E_{0 n 0}$ type must satisfy equation (5) and the following boundary conditions on the perfectly conducting walls of the coaxial volume:

$$
\mathcal{E}(b, t)=\mathcal{E}(a, t)=0 .
$$

The LNMs fields are given by

$$
\begin{aligned}
\mathcal{E} & =A\left[J_{0}\left(\mu_{n} \rho\right) Y_{0}\left(\mu_{n}\right)-J_{0}\left(\mu_{n}\right) Y_{0}\left(\mu_{n} \rho\right)\right] \cos \left(\mu_{n} \tau\right), \\
\mathcal{H} & =-A\left[J_{1}\left(\mu_{n} \rho\right) Y_{0}\left(\mu_{n}\right)-J_{0}\left(\mu_{n}\right) Y_{1}\left(\mu_{n} \rho\right)\right] \sin \left(\mu_{n} \tau\right),
\end{aligned}
$$

where $Y_{m}$ is a Bessel function of the second kind of order $m$, and $\mu_{n}$ is the $n$th positive root of the equation

$$
J_{0}(\beta \mu) Y_{0}(\mu)-J_{0}(\mu) Y_{0}(\beta \mu)=0,
$$

where $\beta=b / a$.

Substituting functions (16) into formulas (41), we obtain an exact solution to the system of equations (1) and (2) in implicit form. It can be verified that the boundary conditions (15) remain valid for the implicit functions $E(r, t)$ and $H(r, t)$ given by (44) and (16). These implicit functions are periodic in time with the period $T_{n}=2 \pi / \omega_{n}$, where $\omega_{n}=\mu_{n}\left(\epsilon_{0} \varepsilon_{1} \mu_{0}\right)^{-1 / 2} a^{-1}$, since the transcendental equations (4) are invariant with respect to the time shifts $\tau \rightarrow \tau+l T_{n}$ with integer $l$. Therefore, equations (4) and (16) describe the fields of NNMs in the coaxial resonator with perfectly conducting walls and a nonlinear nondispersive filling medium.

The fields of NNMs satisfy the initial conditions

$$
E=A\left[J_{0}\left(\mu_{n} \rho e^{\alpha E / 2}\right) Y_{0}\left(\mu_{n}\right)-J_{0}\left(\mu_{n}\right) Y_{0}\left(\mu_{n} \rho e^{\alpha E / 2}\right)\right]
$$

at $t=0$ and $H(r, 0) \equiv 0$, and possess the invariance property.

Let us turn to the results of some calculations by formulas (4) and (16). Figure 1(a) shows the snapshots of 

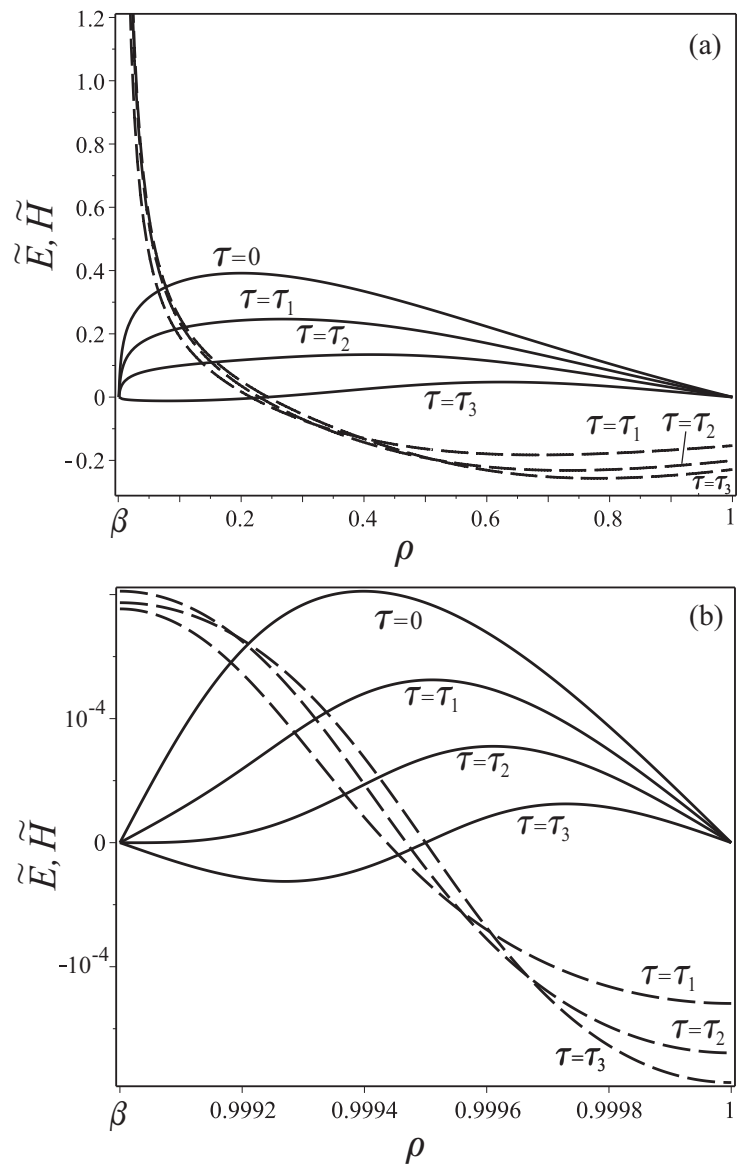

FIG. 1. Electric and magnetic fields as functions of $\rho$ (solid and dashed lines, respectively) in the $n=1$ mode of the coaxial resonator with $\beta=0.001$ (a) and $\beta=0.999$ (b) at times $\tau=0, \tau_{1}=2 \pi /\left(7 \mu_{1}\right), \tau_{2}=2 \pi /\left(5 \mu_{1}\right)$ and $\tau_{3}=\pi /\left(2 \mu_{1}\right)$

the normalized electric and magnetic fields of the NNM of the $E_{010}$ type in the coaxial resonator with $\beta=0.001$ ( $n=1$ and $\mu_{1}=2.65 \ldots$ ) as functions of $\rho$ at fixed instants of time $\tau$. This case corresponds to a thin inner conductor (coaxial wire) inside the cylindrical cavity. For comparison, similar plots for the NNM of the $E_{010}$ type in the cavity with $\beta=0.999\left(\mu_{1}=3141.59 \ldots\right)$ are presented in Fig. 1(b). In the limit $\beta \rightarrow 1$, a coaxial geometry tends to a thin flat layer. Note that Fig. 1 corresponds to the case of strong nonlinearity where $\alpha A=1$.

The presented plots show that the electric fields at different spatial points do not oscillate in unison. However, it is seen in Fig. 1 that the degree of synchronism of the field in the NNM of the $E_{010}$ type inside the cavity turns out to be dependent on the value of $\beta$. The oscillations are closer to synchronous ones for $\beta=0.001$.

The deviations of $E$ and $H$ from their values corresponding to the $E_{010}$ mode in a resonator with $\varepsilon=\epsilon_{0} \varepsilon_{1}=$ const $(\alpha=0)$ for $\beta=0.999$ are more significant than those for $\beta=0.001$, i.e., the nonlinear effects become more pronounced with increasing $\beta$. In the limit $\beta \rightarrow 1$, the eigenvalue $\mu_{n}$ is close to an integer multiple of $\pi$.
This implies the more efficient interaction of harmonics of the eigenfrequency in the spectrum of each NNM. One may say that each NNM is more "internally resonant" in this case.

The total energy of each NNM of the $E_{0 n 0}$ type is again independent of $\alpha$ and exactly coincides with the total energy of the corresponding LNM in the coaxial resonator filled with a linear medium (see the Appendix).

\section{MORE GENERAL OSCILLATIONS. ENERGY ORTHOGONALITY OF NONLINEAR NORMAL MODES}

In this section, we consider more general electromagnetic oscillations which correspond to the presence of an infinite set of NNMs in a cylindrical (noncoaxial) resonator. Let us state the following initial and boundary conditions for the linear wave equation (5):

$$
\begin{aligned}
& \mathcal{E}(\rho, 0)=\Phi(\rho), \quad \frac{\partial \mathcal{E}}{\partial \tau}(\rho, 0)=\Psi(\rho), \quad 0 \leq \rho \leq 1 \\
& \mathcal{E}(1, \tau)=0, \quad|\mathcal{E}(0, \tau)|<\infty, \quad 0<\tau<\infty
\end{aligned}
$$

where $\Phi(\rho)$ and $\Psi(\rho)$ are given functions. The boundary value problem defined by (5), (19), and (20) describes free electromagnetic oscillations with the given initial field distribution in a cylindrical cavity specified by the relations $\rho=r / a \leq 1$ and $0 \leq z \leq L$, which is filled with a linear medium $(\alpha=0)$. The solution to the linear boundary value problem specified by (5), (19), and (20) can be found in a standard way by the method of separation of variables [24]. As a result, the functions $\mathcal{E}$ and $\mathcal{H}$ are written as

$$
\begin{aligned}
& \mathcal{E}(\rho, \tau)=\sum_{n=1}^{\infty} J_{0}\left(\kappa_{n} \rho\right)\left[B_{n} \cos \left(\kappa_{n} \tau\right)+C_{n} \sin \left(\kappa_{n} \tau\right)\right] \\
& \mathcal{H}(\rho, \tau)=-\sum_{n=1}^{\infty} J_{1}\left(\kappa_{n} \rho\right)\left[B_{n} \sin \left(\kappa_{n} \tau\right)-C_{n} \cos \left(\kappa_{n} \tau\right)\right]
\end{aligned}
$$

where

$$
\begin{gathered}
B_{n}=\frac{2}{J_{1}^{2}\left(\kappa_{n}\right)} \int_{0}^{1} \rho \Phi(\rho) J_{0}\left(\kappa_{n} \rho\right) d \rho, \\
C_{n}=\frac{2}{\kappa_{n} J_{1}^{2}\left(\kappa_{n}\right)} \int_{0}^{1} \rho \Psi(\rho) J_{0}\left(\kappa_{n} \rho\right) d \rho .
\end{gathered}
$$

Substituting series (21) into formulas (41), we obtain an exact solution to system of equations (11) and (2) in implicit form. Such an implicit solution describes free electromagnetic oscillations which correspond to the presence of an infinite set of NNMs in the nonlinear resonator. The implicit functions $E(\rho, \tau)$ and $H(\rho, \tau)$ determined by formulas (4) and (21) satisfy the boundary conditions (20), but correspond to somewhat different initial conditions compared with (19). 
For example, let us specify the functions $\Phi$ and $\Psi$ in the simple form

$$
\begin{aligned}
& \Phi(\rho)=A\left(1-\rho^{2}\right), \\
& \Psi(\rho) \equiv 0 .
\end{aligned}
$$

This leads to

$$
B_{n}=8 A \kappa_{n}^{-3}\left[J_{1}\left(\kappa_{n}\right)\right]^{-1}, \quad C_{n}=0 .
$$

At the initial time $\tau=0$, the electric field distribution $E(\rho, 0)$ in the nonlinear resonator is defined by the transcendental equation

$$
E=\sum_{n=1}^{\infty} B_{n} J_{0}\left(\kappa_{n} \rho e^{\alpha E / 2}\right),
$$

while the magnetic field $H \equiv 0$ as in the "seeding" linear problem (see (24) $)$.
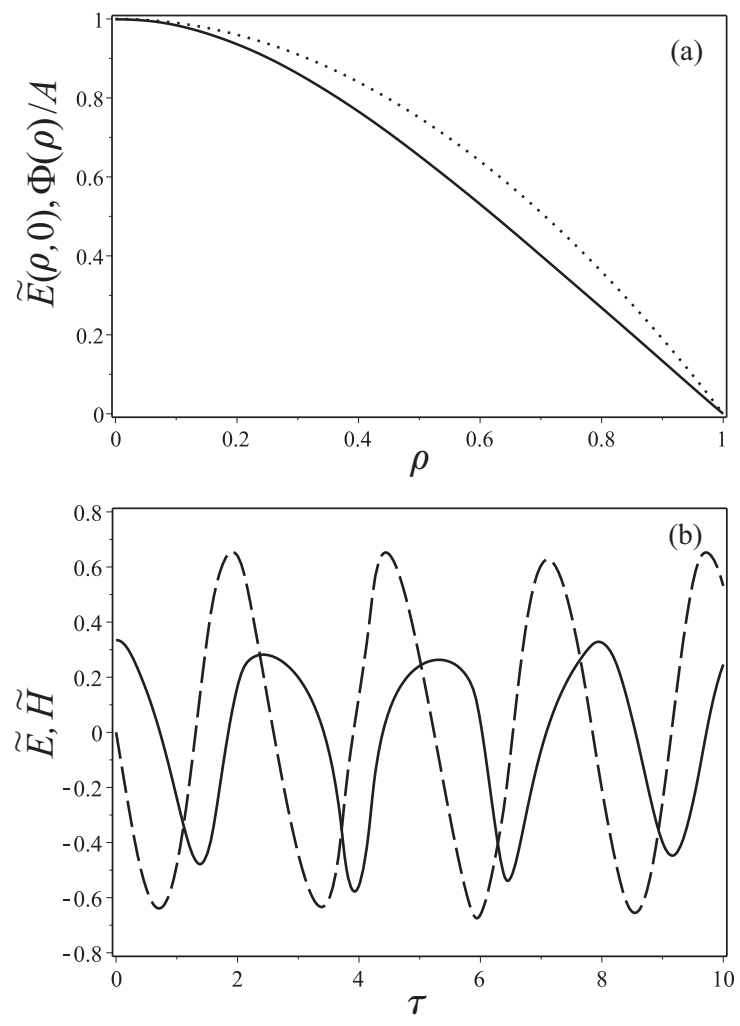

FIG. 2. (a) Initial distribution of the electric field $E(\rho, 0) / A$ (solid line) and the function $\Phi(\rho) / A$ (dotted line). (b) Oscillograms of the electric $(E)$ and magnetic $(H)$ fields of a nonlinear resonator at $\rho=0.75$ (solid and dashed lines, respectively), calculated by formulas (4) and (21) for $\alpha A=0.5$

The electric field distributions $\Phi(\rho) / A$ and $E(\rho, 0) / A$ in the linear and nonlinear cases, respectively, are presented in Fig. 2(a). Figure 2(b) shows oscillograms of the field components $E$ and $H$ at the fixed point $\rho=0.75$ for $\tau>0$. Figure 2 was plotted for $\alpha A=0.5$.

It is important to note that the exact solution obtained for such $\alpha A$ corresponds to single-valued continuous functions $E$ and $H$ (see Fig. 2(b)). While an NNM solution becomes ambiguous with increasing $n$ for any fixed $\alpha A$, the solution yielded by formulas (4) and (21) remains single-valued at moderate $\alpha A$. This fact is stipulated by the rapidly decreasing series coefficients $B_{n}$.

Despite the difference between the initial conditions given by (23) and (26) (see Fig. 2(a)), the energy for the field distribution (23) in the linear cavity resonator $(\alpha=0)$ coincides exactly with the energy of the field defined by (26) in the nonlinear case where $\alpha \neq 0$ (see the Appendix). Due to the energy conservation, the same is true for an arbitrary time instant $\tau>0$. This fact implies a quite remarkable energy orthogonality property of NNMs. The total energy $W$ of the complicated oscillatory process, which is described by exact implicit solution (4), with $\mathcal{E}$ and $\mathcal{H}$ given by (21), is merely the sum of the NNM (or LNM) energies (14):

$$
\begin{aligned}
W & =A^{-2} \sum_{n=1}^{\infty} B_{n}^{2} W_{0}^{(n)}= \\
& =\pi \epsilon_{0} \varepsilon_{1} L a^{2} \int_{0}^{1} \Phi^{2}(\rho) \rho d \rho= \\
& =\frac{\pi}{6} \epsilon_{0} \varepsilon_{1} L a^{2} A^{2} .
\end{aligned}
$$

The performed analysis shows that the energy orthogonality property of the NNMs holds for a rather wide class of initial conditions (19). One should only ensure the convergence of Fourier series (21) and the absence of ambiguity of the implicit solutions.

For the electromagnetic fields governed by the nonlinear system of equations (1) and (2), the principle of superposition does not hold and the NNM fields lack the usual orthogonality property which takes place for linear normal modes in resonators [2]. Moreover, the interaction of the considered NNMs in the forced oscillations can result in complex nonlinear dynamics with a singularcontinuous (fractal) Fourier spectrum [19]. Therefore, the observed energy orthogonality property of the NNMs seems especially interesting.

\section{CONCLUSIONS}

In this work, to the best of our knowledge, we have presented the first nonperturbative approach to the basic properties of NNMs in a distributed nonlinear system. The approach does not require asymptotic expansions and provides a rigorous theoretical formulation of the NNM properties. It is to be emphasized that this formulation is not restricted to consideration of weakly nonlinear systems.

In applying the developed approach, we have constructed exact solutions to the electromagnetic fields of NNMs in cylindrical resonators filled with a nonlinear nondispersive medium. It has been shown that the field oscillations in the found NNMs are periodic in time, but are not synchronous at different spatial points. We have 
established that the total energy of any NNM is independent of the nonlinearity parameter and exactly coincides with the energy of the corresponding LNM in the linear resonator. We have also obtained an exact solution which describes a more general oscillatory process corresponding to the presence of a countable set of NNMs in the nonlinear cylindrical resonator. Based on this solution, we have rigorously established the energy orthogonality property of the NNM fields. A very intriguing and physically important issue, which naturally arises from the present analysis and still remains open, is whether energy orthogonality of NNMs is a property inherent in the particular model of nonlinearity or can be extended to a wider class of nonlinear distributed systems.

\section{ACKNOWLEDGMENTS}

The theoretical study in this work was supported by the Russian Science Foundation (Project No. 1412-00510). Support for the numerical calculations was provided through Contract Nos. 14.B25.31.0008 and 3.1252.2014/K from the Government of the Russian Federation.

\section{APPENDIX}

Let us show that the total energy of free nonlinear oscillations is independent of the nonlinearity parameter $\alpha$ and coincides with the total energy in the linear case $(\alpha=0)$. Due to the energy conservation in a cavity with perfectly conducting walls and a nondispersive filling medium, it is sufficient to prove this fact for an arbitrary fixed time instant (say, $\tau=0$ ). The electric field distribution $E(\rho, 0)$ in the considered nonlinear oscillations is defined by the transcendental equation

$$
E=\mathcal{E}\left(\rho e^{\alpha E / 2}, 0\right),
$$

while $H(\rho, 0) \equiv 0$. Introducing the notation $R=$ $\rho \exp (\alpha E / 2)$, we have

$$
d R=\left(1+\alpha \rho E_{\rho}^{\prime} / 2\right) e^{\alpha E / 2} d \rho .
$$

The derivative $E_{\rho}^{\prime}$ can be found from (A.1) as

$$
E_{\rho}^{\prime}=\left[1-\alpha e^{\alpha E / 2} \rho \mathcal{E}_{R}^{\prime} / 2\right]^{-1} e^{\alpha E / 2} \mathcal{E}_{R}^{\prime} .
$$

Substituting (A.3) into A.2 yields

$$
d \rho=\left[1-\alpha R \mathcal{E}_{R}^{\prime} / 2\right] e^{-\alpha E / 2} d R .
$$

Consider for clarity a cylindrical (noncoaxial) resonator. Making the change of variables and using (A.4), from (7) and (13) one obtains the total energy

$$
\begin{aligned}
W= & 2 \pi \epsilon_{0} \varepsilon_{1} a^{2} L \alpha^{-2} \times \\
& \times \int_{0}^{1}\left(\alpha E+e^{-\alpha E}-1\right) \times \\
& \times\left[1-\alpha R \mathcal{E}_{R}^{\prime} / 2\right] R d R .
\end{aligned}
$$

Here, we have also taken into account the boundary condition $E=0$ at $\rho=1$, implying that $R=1$ for $\rho=1$. It is convenient to rewrite (A.5) as

$$
\begin{aligned}
W= & 2 \pi \epsilon_{0} \varepsilon_{1} a^{2} L \int_{0}^{1}\left[-\frac{1}{2} \mathcal{E} \mathcal{E}_{R}^{\prime} R^{2}+\right. \\
& +\frac{1}{2 \alpha} \mathcal{E}_{R}^{\prime} R^{2}+\frac{1}{\alpha^{2}}\left(e^{-\alpha E}-1\right) R+ \\
& \left.+\frac{1}{\alpha} \mathcal{E} R-\frac{1}{2 \alpha} \mathcal{E}_{R}^{\prime} R^{2} e^{-\alpha E}\right] d R .
\end{aligned}
$$

Integrating the first term in the integrand of (A.6) by parts and using the boundary condition $\mathcal{E}=0$ at $R=1$, we have

$$
-\frac{1}{2} \int_{0}^{1} \mathcal{E}_{R}^{\prime} R^{2} d R=\frac{1}{2} \int_{0}^{1} \mathcal{E}^{2} R d R .
$$

Integrating the second and third terms in the integrand of (A.6) by parts, one can find that the result of integration cancels the last two terms in this integrand. Finally, we get

$$
W=\pi \epsilon_{0} \varepsilon_{1} a^{2} L \int_{0}^{1} \mathcal{E}^{2} R d R
$$

which coincides with the total energy of the linear oscillations. For a coaxial resonator, the proof is similar.

In addition, it should be noted that the total energy of the radially localized field distributions vanishing for $\rho \rightarrow \infty$ in an unbounded nonlinear medium, which can be the case for, e.g., cylindrical electromagnetic waves [18], is also independent of the nonlinearity parameter $\alpha$.
[1] L. D. Landau and E. M. Lifshitz, Mechanics (Pergamon, New York, 1976).

[2] J. D. Jackson, Classical Electrodynamics (Wiley, New
York, 1998).

[3] D. H. E. Dubin and J. P. Schiffer, Phys. Rev. E 53, 5249 (1996). 
[4] A. Paredes, D. Novoa, and D. Tommasini, Phys. Rev. A 90, 063803 (2014).

[5] M. A. Lyapunov, The General Problem of the Stability of Motion (Princeton University Press, Princeton, NJ, 1947).

[6] R. M. Rosenberg, J. Appl. Mech. 27, 263 (1960).

[7] R. M. Rosenberg, J. Appl. Mech. 29, 7 (1962).

[8] R. M. Rosenberg, Adv. Appl. Mech. 9, 155 (1966).

[9] S. W. Shaw and C. Pierre, J. Sound Vib. 169, 319 (1994).

[10] L. I. Manevich and M. A. Pinskii, Int. Appl. Mech. 8, 1005 (1972).

[11] S. W. Shaw and C. Pierre, J. Sound Vib. 164, 85 (1993).

[12] A. F. Vakakis and R. H. Rand, Int. J. Non-Linear Mech. 27, 861 (1992).

[13] A. F. Vakakis, L. I. Manevich, Y. V. Mikhlin, V. N. Pilipchuk, and A. A. Zevin, Normal Modes and Localization in Nonlinear Systems (Wiley, New York, 1996).

[14] W. Lacarbonara, G. Rega, and A. H. Nayfeh, Int. J. Non-Linear Mech. 38, 851 (2003).
[15] G. Kerschen, M. Peeters, J. S. Golinval, and A. F. Vakakis, Mech. Syst. Signal Process. 23, 170 (2009).

[16] M. Peeters, R. Viguié, G. Serandour, G. Kerschen, and J. S. Golinval, Mech. Syst. Signal Process. 23, 195 (2009).

[17] A. V. Kudrin and E. Yu. Petrov, JETP 137, 608 (2010).

[18] E. Yu. Petrov and A. V. Kudrin, Phys. Rev. Lett. 104, 190404 (2010).

[19] E. Yu. Petrov and A. V. Kudrin, Phys. Rev. E 85, 055202(R) (2012).

[20] H. Xiong, L.-G. Si, P. Huang, and X. Yang, Phys. Rev. E 82, 057602 (2010).

[21] V. A. Es'kin, A. V. Kudrin, and E. Yu. Petrov, Phys. Rev. E 83, 067602 (2011).

[22] H. Xiong, L.-G. Si, J. F. Guo, X. Y. Lu, and X. Yang, Phys. Rev. A 83, 063845 (2011).

[23] H. Xiong, L.-G. Si, C. Ding, X. Yang, and Y. Wu, Phys. Rev. E 85, 016606 (2012).

[24] R. Courant and D. Hilbert, Methods of Mathematical Physics (Wiley, New York, 1966). 